\title{
Adverse Drug Reactions of Long-term Intravenous Antibiotics in Patients with Pyogenic Spondylitis
}

\author{
Dong Hwan Kim, Hwan Soo Kim, Kyoung Hyup Nam, Byung Kwan Choi, In Ho Han \\ Department of Neurosurgery, Medical Research Institute, Pusan National University College of Medicine, Busan, Korea
}

Objective: The purpose of this study was to investigate the incidence, cause, and influence of the adverse drug reactions (ADRs) associated with long-term intravenous antibiotics in patients with pyogenic spondylitis (PS).

Methods: We retrospectively reviewed the medical records of 84 patients with PS who underwent intravenous antibiotic therapy in our hospital from January 2001 to December 2012. ADRs were categorized to drug eruption, acute renal failure (ARF), hematologic toxicity, toxic hepatitis, pseudomembranous colitis (PMC), drug fever, and neuronal toxicity. Incidence and onset time of each ADR after antibiotic therapy were analyzed with the incidence of ADRs according to types of antibiotics. Results: ADRs occurred in 38 of the 84 patients (incidence: $45.2 \%$ ). The use of antibiotics was longer in the patients with ADRs (62.7 days) than in the patients without ADRs (44.3 day). The incidence of drug eruption, ARF, hematologic toxicity, toxic hepatitis, PMC, drug fever, and neuronal toxicity were $22.6,11.9,11.9,10.7,7.1,3.6 \%$, and $1.2 \%$, respectively. The duration of antibiotics administration was related to the occurrence of PMC $(p=0.001)$. ADRs were more common in patients treated by glycopeptides including vacomycin and teicoplanin.

Conclusion: The incidence of ADRs due to long-term intravenous antibiotics was as high as $45.2 \%$ in patients with PS. Therefore, we speculate that the possibility of delayed ADRs should be considered after long-term use of the antibiotics. Furthermore, close observation is mandatory to identify and treat ADRs promptly, even though PS revealed the improvement after antibiotic therapy.

Key Words: Spinal extradural abscess · Anti-bacterial agents · Adverse drug reaction

\section{INTRODUCTION}

Pyogenic spondylitis (PS) is the bacterial infection of intervertebral discs and adjacent vertebral bodies ${ }^{2,3,8,12,13)}$. Most of the PS can be treated non-operatively and surgical intervention is inevitable only in $10-20 \%$ of patients ${ }^{3,4)}$. The principles of conservative treatment are to establish an accurate microbiological diagnosis and treat with appropriate antibiotics, immobilize the spine, and closely monitor for clinical and radiographic evidence of spinal instability and progression of in-

- Received: June 9, 2014 - Revised: July 8, 2014

- Accepted: July 15, 2014

Corresponding Author: In Ho Han, MD

Department of Neurosurgery, Pusan National University Hospital, 1-305

Gudeok-Ro, Seo-Gu, Busan 602-739, Korea

Tel: +82-51-240-7257, Fax: +82-51-244-0282

E-mail: farlateral@hanmail.net

*This study was supported by clinical research grant (2013), Pusan National University Hospital

®This is an Open Access article distributed under the terms of the Creative

Commons Attribution Non-Commercial License (http://creativecommons.org/ licenses/by-nc/3.0/) which permits unrestricted non-commercial use, distribution, and reproduction in any medium, provided the original work is properly cited. fection or neurological deterioration ${ }^{18)}$.

Even though the optimal duration of antibiotics therapy is still controversial, long-term intravenous antibiotics at least during 6-8 weeks are essential regardless of the necessity of surgical treatment ${ }^{1)}$. After cessation of the intravenous antibiotics, additional oral antibiotics for 4-8 weeks are administrated according to the patient's response ${ }^{6}$. In addition, approximately half of the recipients receive multiple antibiotics agents. Intravenous antibiotics can cause adverse drug reactions (ADRs). These ADRs can worsen the patient's condition and make it impossible to continue antibiotic treatment. In spite of necessity of long term intravenous antibiotics to the patients with PS, there has been no study about ADRs associated with intravenous antibiotics until now. Thus, the purpose of this study was to investigate the incidence, causes, and clinical outcomes of the ADRs associated with long-term intravenous antibiotics in patients with PS.

\section{MATERIALS AND METHODS}

We retrospectively reviewed the medical records of 84 patients with PS who managed with intravenous antibiotic ther- 
apy in our hospital from January 2001 to December 2012. Due to the retrospective nature, the present study is exempt in accord with the institutional review board standards of our institution. The patients with post-operative spinal infection were excluded from the study. The patients with PS, that is radiologically suspected but organisms have not been isolated, were included from the study.

The patient consisted of 47 men and 37 women with a mean age of $63.2 \pm 12.1$ years. The mean duration of intravenous antibiotic therapy was 52.6 days. By the number of administrated antibiotics, single agent was used in 38 patients (45.2\%). Two, three and four agents were used in 32, 8 and 6 patients, respectively. In 42 of 84 patients, a causative organism was confirmed by tissue cultures or blood cultures.

The incidence and types of ADRs due to intravenous antibiotics were investigated. ADRs were categorized to drug eruption, acute renal failure (ARF), hematologic toxicity, toxic hepatitis, pseudomembranous colitis (PMC), drug fever, and neuronal toxicity. Drug eruption was defined as skin lesions occurred to patients after using antibiotics. Those were maculopapular rashs, puritic lesions, facial edema, urticaria, palpable or extensive purpura, and blisters. A drug eruption due to antibiotics was confirmed by dermatologists. ARF was defined as blood urea nitrogen (BUN) and creatinine increase over normal range and urine volume decreased after using antibiotics. Hepatic toxicity was defined as an abnormal liver function test with elevated aspartate aminotransferase (AST), alanine aminotransferase (ALT), and bilirubin after using anti- biotics. Hematologic toxicity included anemia, leukopenia, or thrombocytopenia which occurred after using the antibiotics. PMC was diagnosed by clinical symptom and laboratory result. Patients with diarrhea more than 3 times per day were suspected to be PMC. PMC was confirmed when the clostridium difficile was isolated from stool culture or positive results in clostridium difficile toxin polymerase chain reaction (PCR).

Incidence and onset time of each ADR after using antibiotics were reviewed and incidence of ADRs according to types of antibiotics was also recorded.

Relation between ADRs and duration of antibiotics administration were evaluated using crossover analysis. In addition, relation between ADRs and confirmation of pathogens were evaluated. Statistical significance was accepted for p-values < 0.05 . SPSS version 12.0 for windows was used for statistical analysis. Categorical variables were analyzed by chi-square test and ANOVA.

\section{RESULTS}

Demographics of patients who underwent antibiotics ther-
Table 1. Clinical data of patients with pyogenic spondylitis

\begin{tabular}{|c|c|c|c|}
\hline Category & All patients & ADRs & No ADRs \\
\hline Number & 84 & 38 & 46 \\
\hline Mean Age (years) & $63.2 \pm 12.1$ & $61.3 \pm 12.7$ & $64.7 \pm 11.5$ \\
\hline SEX (Male:Female) & $47: 37$ & $23: 15$ & $24: 22$ \\
\hline \multicolumn{4}{|l|}{ Number of Antibiotics } \\
\hline 1 & 38 & 17 & 21 \\
\hline 2 & 32 & 16 & 16 \\
\hline 3 & 8 & 4 & 4 \\
\hline 4 & 6 & 1 & 5 \\
\hline \multicolumn{4}{|l|}{ Confirmation of pathogens } \\
\hline Yes & 42 & 16 & 26 \\
\hline No & 42 & 22 & 20 \\
\hline Average treatment duration & 52.6 day & 62.7 day & 44.3 day \\
\hline
\end{tabular}

apy for PS and patients with ADRs were summarized in Table 1.

ADRs occurred in 38 of the 84 patients (incidence: $45.2 \%$ ). The mean age of patients with ADRs was $61.30 \pm 12.7$ years. Men were 23 and female were 15 . In 22 of 38 patients, one ADR occurred, in 13 patients, 2 ADRs occurred, in 2 patients, 3 ADRs occurred and in one patient, 4 ADRs occurred at the same time. The duration of antibiotics therapy was longer in patient with ADRs than patients without ADRs (62.7 days: 44.3 days), and that was statistically significant $(p=0.002)$. But the duration of antibiotic therapy according to confirmation of pathogen did not show significant differences, and that was not statistically significant $(\mathrm{p}=0.883)$.

Incidence, onset time, and treatment of individual ADRs were summarized in Table 2. Incidence of drug eruption, ARF, hematologic toxicity, toxic hepatitis, PMC, drug fever, and neuronal toxicity were 22.6, 11.9, 11.9, 10.7, 7.1, 3.6\%, and $1.2 \%$ in order. Onset time was various for individuals, but ADRs except for PMC mostly occurred at 3-4 weeks after antibiotic use. Mean onset time of PMC was 47.7 days and occurred later compared with other ADRs. Therefore, the duration of antibiotics administration was related to the occurrence of PMC $(\mathrm{p}=0.001)$. Most of ADRs improved after cessation of causative antibiotics or metronidazole administration in PMC, but aminoglycoside-induced ototoxicity was not improved. There was no death due to ADRs caused by antibiotics.

ADRs according to the type of antibiotics were summarized in Table 3. ADRs occurred in 10 of 40 patients treated by $3^{\text {rd }}$ cephalosporin $(25 \%)$, in 17 of 35 patients treated by glycopeptide (48.6\%), and in 3 of 16 patients treated by $1^{\text {st }}$ cephalosporin (18.8\%). Therefore, ADRs were more common in patients treated by glycopeptides including vacomycin and teicoplanin. 
Table 2. Categories of ADRs and their incidence, onset time, and tretement

\begin{tabular}{lccl}
\hline \multicolumn{1}{c}{ ADRs } & Incidence & Mean onset time & Treatment \\
\hline Drug eruption & $19(22.6 \%)$ & 27.6 ( $7-60$ day $)$ & antibiotics change \& topical lotion \\
ARF (acute renal failure) & $10(11.9 \%)$ & $31.9(14-52$ day) & antibiotics stop \& hydration \\
Hematologic toxicity & $10(11.9 \%)$ & $23.0(4-36$ day) & \\
Neutropenia & $6(7.1 \%)$ & $26.5(16-36$ day) & antibiotics stop \\
Thrombocytopenia & $3(3.6 \%)$ & $22.3(12-36$ day) & antibiotics stop \\
Pancytopenia & $1(1.2 \%)$ & 4.0 & antibiotics stop \\
Toxic hepatitis & $9(10.7 \%)$ & $33.0(20-50$ day $)$ & antibiotics stop \& hydration \\
PMC & $6(7.1 \%)$ & $47.7(12-90$ day $)$ & metronidazole or vancomycin \\
Drug fever & $3(3.6 \%)$ & $22.3(19-28$ day) & antibiotics stop \\
Neuronal toxicity Ototoxicity & $1(1.2 \%)$ & 36.0 & antibiotics stop \& steroid \\
\hline
\end{tabular}

Table 3. Incidence of ADRs according to types of antibiotics

\begin{tabular}{lc}
\hline \hline Causative antibiotics & Incidence of ADRs \\
\hline $3^{\text {rd }}$ cephalosporin & $10 / 40(25.0 \%)$ \\
Glycopeptide & $17 / 35(48.6 \%)$ \\
$1^{\text {st }}$ cephalosporin & $3 / 16(18.8 \%)$ \\
Quinolone & $2 / 12(16.7 \%)$ \\
Aminoglycoside & $2 / 11(18.2 \%)$ \\
Penicillin & $2 / 7(28.6 \%)$ \\
Carbapenem & $1 / 4(25.0 \%)$ \\
Lincosamide & $2 / 3(66.7 \%)$ \\
Unknown & 3 \\
\hline
\end{tabular}

Unknown: not to confirm causative one among multiple antibiotics

\section{DISCUSSION}

Since the advent of antibiotics, the mortality rate of pyogenic spondylitis has been reported as less than $5 \%^{5,11,14)}$. Despite advance in antibiotics, 6 to $7 \%$ of hospitalized patients still experience serious ADRs due to antibiotics. In the United States, 100,000 die of serious ADRs due to antibiotics annually ${ }^{7}$. Although long-term intravenous antibiotics have been used as a formal treatment of pyogenic spondylitis, there was no study about these ADRs in patients with PS. Therefore, we focused on the ADRs associated with long-term antibiotics for PS.

The incidence of ADRs associated with antibiotics was as high as $45.2 \%$ in the patients with PS. In addition, the duration of treatment in the patients with ADRs was longer than that in the patients without ADRs and ADRs due to antibiotics may affect prognosis and medical cost of the patients. Therefore, we suggest that spine surgeon should explain the possible ADRs associated with antibiotic therapy and cessation of antibiotics therapy.
Interestingly, our study showed that the duration of treatment was longer in the patients with $\mathrm{ADRs}$ (62.7 days) than in the patients without $\mathrm{ADRs}$ (44.3 day). If ADRs occur during treatment of pyogenic spondylitis, antibiotics should be stopped and changed or the dosage of antibiotics may be reduced until ADRs improve. We assume that the duration of treatment was elongated for these reasons.

The most common ADR was drug eruption showing incidence of $22.6 \%$. Drug eruption was not serious and completely improved after antibiotics change and topical lotion. However, more serious ADRs such as ARF, hematologic toxicity and toxic hepatitis showed relatively high incidence over $10 \%$. Fortunately, those ADRs were completely improved without mortality or morbidity after antibiotics cessation and medical treatment.

Among the ADRs, PMC and drug fever may be carefully considered during treatment of PS. In most of PS, C-reactive protein (CRP) and fever gradually decrease after appropriate antibiotics use. However, if PMC or drug fever occurs during antibiotics therapy, rebounded CRP and fever can mask the effectiveness of the antibiotics for PS. Therefore, when CRP or fever rebounds during antibiotic therapy, spine surgeons should keep in mind the possibility of PMC or drug fever. Especially, the symptoms of PMC are broad, including from mild symptoms, a few diarrheic episodes and no fever, to threatening fulminant colitis or toxic megacolon ${ }^{15,16}$. Therefore, early diagnosis and treatment is important and stool culture is the gold standard for diagnosis, with sensitivity close to $100 \%$. The detection of toxin in stool culture is less sensitive than traditional stool culture and its sensitivity is 70\%. The most used laboratory methods are immune enzyme assays, with results in up to 2 hours. Another highly sensitive and specific method is PCR and colonoscopy can be performed to diagnose quickly if needed ${ }^{17)}$.

In our patients, most of ADRs improved after timely treat- 
ment and no patient expired by ADRs, but in a patient, permanant hearing loss remained due to amnoglyocide-induced ototoxicity. As there is no definite treatment regimen about ototoxicity, prevention is very important ${ }^{9)}$. Therefore, routine audiography be sometimes recommended in patients given potentially ototoxic agents, but, in practice, such examination test is not routinely conducted ${ }^{7,19)}$.

In our study, onset time of ADRs was various from 4 days to 90 days, but most of ADRs except for PMC occurred at 3-4 weeks after antibiotic therapy. PMC appeared at 6 weeks after antibiotic therapy. Based on our results, meticulous care is required to identify and respond quickly to ADRs at 3-4 weeks, even though PS clinically improves after antibiotic therapy. In addition, in order to reduce undesirable outcomes associated with ADRs, doctors should try to reduce the inappropriate and excessive use of antibiotics and treatment duration. Generally, to reduce the duration of antibiotic therapy, it is thought that accurate microbiological diagnosis by culture and selective antibiotic use may be important. But in our case, the duration of antibiotic therapy and the incidence of ADRs according to confirmation of pathogen did not show significant differences. Lee et al. stated that negative culture results did not significantly affect the clinical course of pyogenic spondylodiscitis when we compared the clinical courses of both groups ${ }^{10)}$.

Four types of antibiotics, $1^{\text {st }}$ cephalosporin, $3^{\text {rd }}$ cephalosporin and glycopeptides are commonly used in pyogenic spondylitis. Therefore, ADRs by those antibiotics were also common. Especially, ADRs due to glycopeptides are more common and doctor should be care about occurrence of ADRs after glycopeptides use.

\section{CONCLUSION}

The incidence of ADRs due to long-term intravenous antibiotics was as high as $45.2 \%$ in patients with PS. These ADRs can affect prognosis of the patients and increase the duration of hospital stay and medical cost. Therefore, we suggest that doctors should sufficiently explain the possible ADRs to patients and their family members at the beginning of antibiotics therapy. Close observation is also required to identify and treat ADRs early, even though PS clinically improves after antibiotic therapy.

\section{REFERENCES}

1. Bettini N, Girardo M, Dema E, Cervellati S: Evaluation of con- servative treatment of non specific spondylodiscitis. Eur Spine J 18 Suppl 1:143-150, 2009

2. Butler JS, Shelly MJ, Timlin M, Powderly WG, O’Byrne JM: Nontuberculous pyogenic spinal infection in adults: A 12-year experience from a tertiary referral center. Spine (Phila Pa 1976) 31(23):2695-2700, 2006

3. Cheung WY, Luk KD: Pyogenic spondylitis. Int Orthop 36(2): 397-404, 2012

4. Cottle L, Riordan T: Infectious spondylodiscitis. J Infect 56(6): 401-412, 2008

5. Friedman JA, Maher CO, Quast LM, McClelland RL, Ebersold MJ: Spontaneous disc space infections in adults. Surg Neurol 57(2):81-86, 2002

6. Gouliouris T, Aliyu SH, Brown NM: Spondylodiscitis: Update on diagnosis and management. J Antimicrob Chemother 65 Suppl 3:iii11-24, 2010

7. Granowitz EV, Brown RB: Antibiotic adverse reactions and drug interactions. Crit Care Clin 24(2):421-442, xi, 2008

8. Griffiths HE, Jones DM: Pyogenic infection of the spine. A review of twenty-eight cases. J Bone Joint Surg Br 53(3):383-391, 1971

9. Guthrie OW: Aminoglycoside induced ototoxicity. Toxicology 249(2-3):91-96, 2008

10. Koo SK, Hwang BW, Lee SH: Anterior lumbar interbody fusion with percutaneous pedicle screw fixation for the treatment of postoperative pyogenic spondylodiscitis. Korean Journal of Spine 7(4):276-279, 2010

11. Legrand E, Flipo RM, Guggenbuhl P, Masson C, Maillefert JF, Soubrier M, et al: Management of nontuberculous infectious discitis. Treatments used in 110 patients admitted to 12 teaching hospitals in france. Joint Bone Spine 68(6):504-509, 2001

12. Lehovsky J: Pyogenic vertebral osteomyelitis/disc infection. Baillieres Best Pract Res Clin Rheumatol 13(1):59-75, 1999

13. Maiuri F, Iaconetta G, Gallicchio B, Manto A, Briganti F: Spondylodiscitis. Clinical and magnetic resonance diagnosis. Spine (Phila Pa 1976) 22(15):1741-1746, 1997

14. McHenry MC, Easley KA, Locker GA: Vertebral osteomyelitis: Long-term outcome for 253 patients from 7 cleveland-area hospitals. Clin Infect Dis 34(10):1342-1350, 2002

15. Pepin J, Valiquette L, Alary ME, Villemure P, Pelletier A, Forget $\mathrm{K}$, et al: Clostridium difficile-associated diarrhea in a region of quebec from 1991 to 2003: A changing pattern of disease severity. CMAJ 171(5):466-472, 2004

16. Pepin J, Valiquette L, Cossette B: Mortality attributable to nosocomial clostridium difficile-associated disease during an epidemic caused by a hypervirulent strain in quebec. CMAJ 173(9): 1037-1042, 2005

17. Silva Junior M: Recent changes in clostridium difficile infection. Einstein (Sao Paulo) 10(1):105-109, 2012

18. Skaf GS, Domloj NT, Fehlings MG, Bouclaous CH, Sabbagh AS, Kanafani ZA, et al: Pyogenic spondylodiscitis: An overview. J Infect Public Health 3(1):5-16, 2010

19. Xie J, Talaska AE, Schacht J: New developments in aminoglycoside therapy and ototoxicity. Hear Res 281(1-2):28-37, 2011 